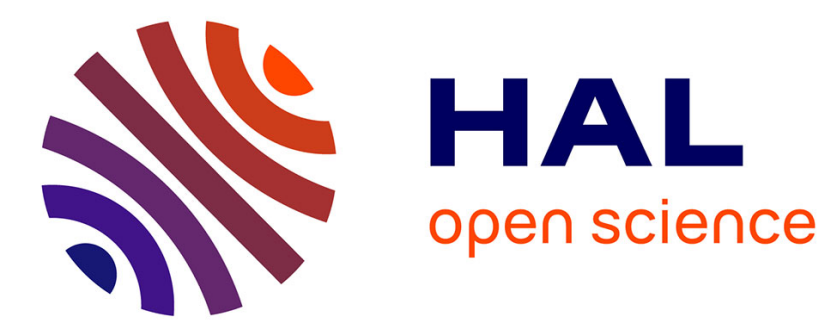

\title{
Possibility spaces and the notion of novelty: from music to biology
}

\author{
Maël Montévil
}

\section{To cite this version:}

Maël Montévil. Possibility spaces and the notion of novelty: from music to biology. Synthese, 2019, 196 (11), pp.4555-4581. 10.1007/s11229-017-1668-5 . hal-02398774

\section{HAL Id: hal-02398774}

\section{https://hal.science/hal-02398774}

Submitted on 8 Dec 2019

HAL is a multi-disciplinary open access archive for the deposit and dissemination of scientific research documents, whether they are published or not. The documents may come from teaching and research institutions in France or abroad, or from public or private research centers.
L'archive ouverte pluridisciplinaire HAL, est destinée au dépôt et à la diffusion de documents scientifiques de niveau recherche, publiés ou non, émanant des établissements d'enseignement et de recherche français ou étrangers, des laboratoires publics ou privés. 


\title{
Possibility spaces and the notion of novelty: from music to biology
}

\author{
Maël Montévila,b,* \\ ${ }^{a}$ Laboratoire "Matière et Systèmes Complexes" (MSC), UMR 7057 CNRS, Université Paris 7 Diderot, Paris, France \\ ${ }^{b}$ Institut d'Histoire et de Philosophie des Sciences et des Techniques (IHPST) - UMR 8590, 13, rue du Four, 75006 Paris, France
}

\begin{abstract}
We provide a new perspective on the relation between the space of description of an object and the appearance of novelties. One of the aims of this perspective is to facilitate the interaction between mathematics and historical sciences. The definition of novelties is paradoxical: if one can define in advance the possibles, then they are not genuinely new. By analyzing the situation in set theory, we show that defining generic (i.e., shared) and specific (i.e., individual) properties of elements of a set are radically different notions. As a result, generic and specific definitions of possibilities cannot be conflated. We argue that genuinely stating possibilities requires that their meaning has to be made explicit. For example, in physics, properties playing theoretical roles are generic; then, generic reasoning is sufficient to define possibilities. By contrast, in music, we argue that specific properties matter, and generic definitions become insufficient. Then, the notion of new possibilities becomes relevant and irreducible. In biology, among other examples, the generic definition of the space of DNA sequences is insufficient to state phenotypic possibilities even if we assume complete genetic determinism. The generic properties of this space are relevant for sequencing or DNA duplication, but they are inadequate to understand phenotypes. We develop a strong concept of biological novelties which justifies the notion of new possibilities and is more robust than the notion of changing description spaces. These biological novelties are not generic outcomes from an initial situation. They are specific and this specificity is associated with biological functions, that is to say, with a specific causal structure. Thus, we think that in contrast with physics, the concept of new possibilities is necessary for biology.
\end{abstract}

Keywords: Novelty, Possibility space, Biological functions, Organization, Emergence

\section{Introduction}

The theory of evolution assumes that current life forms are the result of variations of preceding life forms. Since past life forms did not have all the features of current ones, it is necessary to think that novelties appear (and disappear) in the process of evolution. As a result, developing this theory has immediately led to ponder on biological novelties, and both Lamarck and Darwin discuss them (Muller \& Wagner, 1991). The current phylogenetic classification of living beings uses the concept of novelty as a way to estimate the genealogical relationship between taxa. For example, phylogenetic trees minimize the number of novelty appearances to maximize the coherency of the classification. In general, the concept of open-ended evolution is central to biology, and some authors even use this notion to define living systems (Ruiz-Mirazo et al., 2004).

\footnotetext{
${ }^{*}$ Corresponding author

Email address: mael .montevil@gmail.com (Maël Montévil)

$U R L:$ http://montevil.theobio.org (Maël Montévil)

- Published as: Montévil, M. Possibility spaces and the notion of novelty: from music to biology Synthese (2018). https://doi.org/10 1007/s11229-017-1668-5
}

However, the mathematical modeling of novelties has been more neglected. For example, population genetics usually describe abstract traits and their consequences on fitness. In this field, the space of possibilities is therefore limited to allele frequencies and phenotypic novelties, if any, are postulated, not explained. By contrast, the artificial life community is struggling to provide computational frames displaying open-ended evolution, where "open-ended" is an ambiguous concept which embeds some idea of generating novelties. "The particular properties that characterize open-ended evolution are tricky to pin down and often lack consensus [...]. Yet despite the difficulty of precisely pinpointing this phenomenon, a major goal of artificial life (alife) research remains to observe open-ended evolution in an alife simulation (Bedau et al., 2000). In fact, there is little doubt that no algorithm yet devised has fully reproduced it." (Soros \& Stanley 2014) There is an intuitive reason why this goal of alife is challenging and more generally why there is a tension between mathematics and the notion of novelty. In mathematics, the structure of logical proofs is hypothetic-deductive, meaning that there should be nothing genuinely new in the proof after the hypotheses have been formulated. The same applies, mutadis 
mutandis, to computational frameworks.

Let us consider a few definitions of novelty in evolutionary biology. Most of these definitions discriminate the relevant novelties from the irrelevant ones on the basis of a given theoretical perspective, but they do not expand on the newness of novelties per se. For example, Mayr proposes a definition focused on adaptation where biological novelties are "any newly acquired structure or property that permits the performance of a new function, which, in turn, will open a new adaptive zone" (Mayr 1963). Other definitions emphasize development: "Ian evolutionary novelty is] a novel trait [based on] a qualitatively distinct developmental variant" (West-Eberhard 2003). These definitions aim to discuss what are the biologically relevant novelties according to a given theoretical perspective. We agree that this is an aspect of the problem. However, these definitions are mostly tautological concerning what it means for something to be new. A self-contained notion of novelty should intrinsically define being new. Muller \& Wagner (1991) provide a more precise definition by stating that "a morphological novelty is a structure that is neither homologous to any structure in the ancestral species nor homonymous to any other structure of the same organism." Here, the concept of novelty is defined by the heterogeneity with respect to a history and the rest of the organisms considered. This notion has lead to a specific research program which defines novelty by development (Wagner \& Lynch, 2010). However, novelties associated with the functioning of organisms and a fortiori functions are then excluded. Moreover, this notion cannot be used straightforwardly in the mathematical thinking on novelties which, we argue, is a more general problem.

Emergence is a philosophical concept that is relevant for novelty. Typically, emergence corresponds to two notions that are analytically distinct. The first is synchronic emergence which is concerned with the irreducibility of a system to the analysis of its components when they are in isolation (or in simpler systems). The second, diachronic emergence, is of direct interest here because it is defined by the notion of novelty (Stephan, 1999; Bich \& Bocchi, 2012). Diachronic emergence typically comes in different variants depending on the predictability and reducibility of novelties from the initial state of affairs.

Let us consider a few physical situations which can be interpreted as modeling the appearance of novelties and are regarded as models of emergence (Anderson, 1972; Anderson \& Stein, 1985). Novelty in physics cannot just be the appearance of a specific configuration that never appeared before. For example, it is clear that the microscopic state (position and momentum of all particles) of a gas at equilibrium in a room is new in the sense that the odds of it occurring twice in the universe's lifetime are vanishingly small. However, as far as equilibrium thermodynamics is concerned, this precise state does not represent something new: the macroscopic description of the gas will match those at other time points and is stationary for all intents and purposes. By contrast, the formation of a crystal from a liquid or a gas involves the appearance of patterns corresponding to the directions of the periodic disposition of atoms or molecules. These "new" patterns play a theoretical and causal role since they explain why crystals do not have the same mechanical and electrical properties in all directions, unlike gases and liquids. Think for example of graphite which tends to break along specific directions or crystals which tend to have facets. In these situations, macroscopic structures that were not present in the initial conditions appear and are theoretically meaningful. Diachronic emergence is also relevant for chaotic dynamical systems where the unpredictability of the outcome is the matter of philosophical interest (Stephan, 1999).

These models of physical phenomena are defined by stable equations and space of possibilities; however, several authors, including myself, argue that these assumptions are inadequate for biology and propose alternative viewpoints. Recent theoretical works study the consequences of novelty and argue that biology requires a framework for changes of possibility space (Kauffman, 2002, Longo \& Montévil, 2011, 2013a, 2014, Longo et al., 2012; Montévil et al., 2016; Loreto et al., 2016; de Vladar et al., 2017) and that the same applies to economy (Koppl et al., 2015; Kauffman, 2016). From a philosophical perspective, the issue pertains both to emergence (Bich \& Bocchi, 2012) and process philosophy (Koutroufinis, 2014, 2017). In these approaches, the object is not well described by an invariant mathematical space. Instead, the objects require that mathematical spaces change over time ${ }^{1}$ More generally, invariant mathematical structures do not define these theoretical frameworks; instead, they aim to accommodate changing mathematical structures. One aim of these frameworks is to accommodate biological novelties. However, an explicit analysis of the concept of novelty in this context is still required and this paper precisely aims to perform such an analysis.

In this paper, we discuss the notion of new possibilities and some of its conceptual challenges. In particular, we aim to provide a framework to respond to typical objections by mathematicians and physicists. These objections correspond to the following line of reasoning. We can always define spaces that are large enough to seemingly accommodate every possibility so that there is no need for the concept of new possibility and the concept of possibility is sufficient. For example, spaces of all possible forms should be able to accommodate all biological shapes, or spaces of all possible mathematical functions should be sufficient to model any biological interactions. This reasoning enables physicists and mathematicians to think about the situation in the hypothetic-deductive

\footnotetext{
${ }^{1}$ The mathematical space used to describe an object is the combination of all the quantities that are used to describe its state. For example, a cell population can be described by the number of cells $n$ and the corresponding mathematical space is then the positive integers.
} 
framework that we mentioned in the beginning of this section. In practice, the spaces of possibilities used are far smaller but remain static, for example, in physical approaches to evo-devo (Zhu et al., 2010). In another context, this line of reasoning leads to the historical thought experiment considering the set of all books of a given length. This idea has been discussed by Leibniz (1991, p.61) and popularized by Borges (1998) under the name of the Library of Babel. Such a construct seems to exclude the notion that human arts and sciences generate new books and that paradigm shifts entail new possibilities for books.

To gain a better understanding of the concept of novelty and of new possibilities, we start from a paradox that stems from Bergson's work. Bergson discusses the case of symphonies and states that a symphony is not possible before it becomes real because conceiving the precise possibility of a symphony is equivalent to composing it. However, we point out that one can define the set of all possible music scores as the set of combinations of musical symbols. We show that the confrontation of these two lines of reasoning leads to a paradox. We then argue that the concepts of possibility and of novelty require a more precise discussion than a set theoretical definition. Defining generically the elements of a set is not the same thing as defining the individual properties of each of its elements. In a second part, we apply this discussion to biology. We show that the notion of new possibilities is relevant even from perspectives that seem incompatible with it, such as genetic determinism. We characterize the notion of novelty in physical models of self-organization and conclude that they do not require new possibilities. We then elaborate on biological novelties and argue that new possibilities are relevant. We will also show that novelties associated with biological functions have a special theoretical role.

\section{New possibilities: an enlightening paradox}

Several authors have recently emphasized the need to take into account changes of the space of description of biological objects (Kauffman, 2002; Longo \& Montévil, 2011, 2013a, 2014; Longo et al., 2012; Montévil et al., 2016; Loreto et al., 2016). In Montévil et al. (2016), we argue that this assumption is part of a fundamental theoretical principle: the mathematical space required to describe and understand the organization of an organism may change with the flow of time, both in life cycles and over evolutionary time scales. In these frames, changes of possibility space are a counterpart to the qualitative changes of biological objects which, in evolutionary theory, lead to the remarkable diversity of current life forms. This perspective is foreign to physics where the possibility space is always postulated as an $a$ priori of the theoretical description.

In this section, we will discuss in greater details the concept of new possibilities. This concept is a core com- ponent of Bergson's philosophy of time. It underlies the philosophical understanding of the creativity of living beings in evolution. We will use the following text to show a paradox that helps to understand new possibilities.

When a musician writes a symphony, was his work possible before it became real? Yes, in the sense that there was no insurmountable obstacle to realize it. However, it is easy to shift from this entirely negative meaning of the word to a positive one without noticing it: one pictures that everything that happens may be perceived beforehand by a sufficiently informed mind, and thus preexist in an ideal form to its realization; - this idea is absurd in the case of a work of art since as soon as the musician has a precise and complete idea of the symphony he is going to produce, the symphony is done. Neither in the mind of the artist nor, a fortiori, in any other mind comparable to ours, even impersonal or merely virtual, would the symphony lay as a possibility before it became real. (Bergson 2014, we translate.)

We think that this statement of Bergson leads to a paradox and that this paradox is key to a better understanding of the concept of novelty. The paradox is that it is possible to define a set which includes all written symphonies, thus arguably all possible symphonies, and, at the same time, that there is no obvious flaw in Bergson's reasoning. Can we define the possibility of a symphony without composing it?

There is a standardized way to write classical music and the writing of a symphony leads to a music score: a finite sequence of symbols from a finite set of symbols (the notes and their kinds). Let us call $M_{s}$ the set of music scores for a given set of instruments. $M_{s}$ is a countable set, and the set of all possible symphonies seems to be mathematically well-defined. $M_{S}$ is based on the same principle as Leibniz (1991) and Borges (1998) idea of a library containing all books of a finite, given length in our alphabet. We are aware that some composers use extended writing systems, that new musical instruments get continually invented, and that the symphony is not just its music score. However, we focus on the difficulty raised by the concept of novelty when a space of description is well-defined, which is the situation that seems the most opposed to a strong notion of novelty.

Let us now phrase our paradox. By defining $M_{s}$, it seems that we define all possible symphonies. At the same time, Bergson's reasoning has no obvious flaw: having a precise and complete idea of the possibility of a symphony implies that this symphony has already been composed. To solve the discrepancy between these two line of reasoning, we will argue that defining $M_{s}$ is very 
different from defining musically relevant symphonies. The core of our argument is the distinction between the set $M_{s}$ of possible music scores defined by a writing system and the putative set of possible symphonies endowed with an assessment of their musical quality.

\subsection{Defining a set differs from defining each of its elements individually}

In the following discussion, we carefully analyze the meaning of defining a possibility. We want first to introduce a conceptual distinction between mathematical objects defined collectively, in a generic manner, and the actual definition of an individual or specific element.

An example will show why this distinction is mandatory in mathematics. In a given logical axiomatic, the possible definitions that one can produce form a countable set: the possible definitions are as numerous as natural numbers: they are finite combinations of symbols from a finite set of symbols. However, the set of real numbers is not countable; it has a larger cardinality than natural numbers. In this sense, there are far more real numbers than usable definitions of specific real numbers. The real numbers that can be defined individually, such as $\pi$ or $\frac{1}{2}$, are very few in comparison with the ones that cannot be defined individually. Actually, the probability of being able to define specifically a real number chosen randomly $\sqrt{2}$ is zero. Another way to emphasize this point is to say that there are more real numbers that possible names to name them individually, which makes most individual real numbers ineffable. 3 Real numbers can still be defined, for example using the Dedekind cut, but this definition is a generic one. Thus, defining a set of possibilities generically and individually defining each one of its elements are very different notions.

The lack of specific definitions for each real numbers does not prevent mathematical reasoning on them. Instead of reasoning on specific numbers, in most cases, reasoning involves generic properties where numbers appear as generic variables. For example, the statement $x^{2} \geq 0$ is valid for any real number $x$ and this statement is about a generic $x$. Any given set of axioms enables mathematicians to discuss only certain properties which are the properties of a few individual cases and generic properties.

Since only finite proofs are possible, it is only possible to handle a finite number of specific cases. As a result, reasoning on infinite sets requires generic statements. For example, induction on natural numbers consists in the proof of a generic formula $\mathscr{P}(n)$ for $n=0$ (or

\footnotetext{
${ }^{2}$ Here randomly means, for example, a number chosen randomly in a finite interval with the uniform probability distribution.

${ }^{3}$ The axiom of choice illustrates this point. The axiom of choice enables the mathematician to pick specific numbers without an explicit method to do so. In this sense, the action of choosing a specific number becomes generic. An axiom is required for this operation because such a method cannot be constructed.
}

any finite number of individual cases) and then on a proof that for a generic $n, \mathscr{P}(n)$ implies $\mathscr{P}(n+1)$. Then, the axiom of induction states intuitively that the validity of $\mathscr{P}$ is "propagated" from $n=0$ to all $n$. Note that in the case of natural numbers, unlike real numbers, every number can be defined individually, by counting for example. Nevertheless, it is never possible to actually define all natural numbers individually because there is an infinity of them: counting has no end.

From the viewpoint of mathematical logic, we thus have three different situations for the definition of individual properties:

- The set is defined, but not all of its elements can be defined individually within any axiomatic, like in the case of the real numbers.

- The set is defined, and all individual elements can be defined in principle. However, any actual discussion can only involve a subset of individual elements because the set is infinite. A paradigmatic example is the set of natural numbers.

- The set is finite, and there is no principled limitation.

Let us now go back to symphonies. The definition of the set of music scores $M_{s}$ is a generic definition. The paradox that we exposed stems from the idea that defining $M_{s}$ would be enough to define all possible symphonies so that $M_{s}$ would include the writing of any symphony before it is composed. However, we have shown that conflating the generic definition of a set and the individual definitions of its elements is not logically correct. In Bergson's words, defining a set does not always provide a "precise and complete idea" of all its individual elements. Now, does this line of reasoning applies to symphonies? $M_{S}$ is countable since music scores are finite combinations among a finite number of symbols. Therefore, $M_{s}$ is comparable to natural numbers and all individual music score can be defined by a finite axiomatic, even though only a few of them can be defined in any discussion. However, is this sufficient to define all the possible symphonies?

\subsection{The relevant properties are what matters}

To state the possibility of a symphony, we think that it is necessary to check at least that the putative symphony is an admissible symphony and not just any sequence of symbols. Here, "symphony" means loosely a musical piece that a music lover enjoys. ${ }^{4}$ The set of music scores which are symphonies would be a subset of all possible music scores $M_{s}$. The issue lies in the definition of this

\footnotetext{
${ }^{4}$ For example, this notion could be implemented in a similar way than Turing's imitation game (Turing, 1950), with listeners deciding whether a piece is admissible.
} 
set. Even in mathematical logic, problems in the definition of a set are not circumscribed to the definition of individual elements. The issue is sometimes to decide whether elements are part of the set or not. For example, the definition of subsets of natural numbers may require far more complex logics than the definition of natural numbers themselves. Defining a subset is a problem that also appears when the definition depends on the world. For example, the set of the couples (year, French president elected) are a subset of numbers $\times$ string of characters. However, the number of elements of this set that we can enumerate depends on when we are performing this enumeration.

In sciences, properties which have an explanatory role are central, and it should be the same when defining possibilities and novelties. In physics, it is usual for relevant properties to be generic. For example, the force exerted on an object $A$ in free fall is $m \vec{g}$ where $\vec{g}$ is the gravity field, and $m$ is the mass of $A$. The analysis of free fall does not depend on the individual values of $m-$ being real numbers most individual masses are ineffable - or on the nature of the object. Instead all possible $m$ lead to the same analysis of trajectories. The genericity of the analysis is possible because physics is not about quantities. Instead, physics is based on (generic) relations between quantities.

In general, dynamical systems are analyzed for generic values of their parameters, with possible punctual bifurcation points corresponding to qualitative changes in the dynamics. Similarly, physicists analyze generic initial conditions. Sets of initial conditions lead to the same qualitative dynamics, and these sets are called the basin of attraction of this qualitative dynamics.

These qualitative changes are another example of our discussion in the previous section: mathematics can treat a finite number of individual cases and an infinite number of generic cases. In some situations, there is a finite number of bifurcations, and a discussion of every individual case is possible. In other situations, the number of bifurcations is infinite, but their process is generic which makes an exhaustive analysis possible. We will expand on this point as it illustrates the plasticity of reasoning in terms of genericity and ultimately the importance of this concept.

We will consider the paradigmatic example of the period doubling scenario in the case of the logistic map. These dynamics depend on a parameter $r$ with values between 1 and 4 . For $r$ between 1 and 3, the trajectory tends towards a single point $((r-1) / r)$. For $r$ between 3 and $1+\sqrt{6}$, the dynamics tends to oscillate between two different values. For now, we have two sets of generic situations, which can be analyzed individually and which correspond to qualitatively different behaviors. Now the situation becomes more complicated as $r$ tends towards $r_{c} \approx 3.56 \ldots$ since the system undergoes a cascade of bifurcations where each bifurcation corresponds to a doubling of the period of the trajectories. There is an infinite number of bifurcation when we increase $r$ till $r_{c}$. We cannot analyze an infinite number of situations individually, but physicists and mathematicians point out that all these bifurcations are actually more of the same: they correspond to a doubling of the period. To discuss the situation, they analyze the generic process of period doubling when $r$ becomes close to $r_{c}$, and this leads to relevant predictions (Feigenbaum, 1980). The situation is analogous to the analysis of fractals: fractals look heterogeneous with qualitative patterns at all scales, but all scales are symmetric and a generic analysis is then possible.

This discussion applies mutadis mutandis to probabilistic models. In these models, sets of possibilities are endowed with probabilistic weights which are used to analyze the intended phenomena. Sets of possibilities with probability 0 are considered irrelevant, they are not forbidden but never happen in practice and thus do not play a theoretical role. As a result, discussing their specific properties is not required. Mathematicians call "almost sure" the properties which are met in all cases except for a subset of probability 0 . Being almost sure is a form of genericity which aims to disregard irrelevant qualitative cases. For example, in statistical mechanics, the probability of a configuration with an entropy below the maximum is 0 . Thus, only some macroscopic possibilities are relevant ${ }^{5}$ Let us emphasize this point. In statistical mechanics, all microscopic states are possible. These possibility spaces include all kinds of patterns that are remarkable for a human observer. For example, some molecules may happen to be aligned in a gas at a given time. These patterns may even include letters and words. However, these patterns are purely accidental, they are not sustained, and they do not play a particular causal role. Instead of discussing these patterns, the theory focuses on generic properties. These generic properties are robust and enable physicists to ultimately restrict the discussion to relatively simple equations such as the ones of thermodynamics. In general, physical models and theories restrict the discussion to generic properties and do not have to examine the specific properties that some individual microscopic states display.

By contrast, we think that the relevant properties of symphonies are not generic or at least they are not generic properties of the set $M_{S}$ of music scores. Indeed, all music scores do not make sense as symphonies. There are attempts to consider generic properties of a given musical style (usually the style of a specific author or interpret) and then to generate new music scores or soundtracks verifying these generic properties (Pachet \& Roy, 2014, Papadopoulos et al., 2016). These attempts use machine learning in combination with a few generic criteria that musical patterns are assumed to follow. The aim is

\footnotetext{
${ }^{5}$ This is valid, for example, in a situation without energetic constraints and with an infinite number of particles.
} 
to obtain a generic generator of acceptable soundtracks and thus to define and explore sets that have generic regularities that are assumed to be musically relevant. However, these generic regularities are not written in the algorithm, and they are not pre-stated (except for the generic criteria mentioned above). Instead, they are extracted by machine learning. Thus, the individual works of the musician rigorously preexist the definition of the generic properties extracted from machine learning and not the other way around. It follows that these generic sets are specific to the past of an individual composer or style and are subordinated to it. Moreover, assuming that there can be genuine qualitative novelties that result from probing these sets, there is no guaranty that they would be musically interesting.

Let us now conclude on this part. In physics (and epistemologically similar modeling approaches), the relevant properties are usually generic properties. Physicists understand systems whose states are in enormous sets thanks to these generic properties and not by the specific properties of the individual possibilities (the elements of these sets). In the case of music, the set of possible music scores differs from the set of possible symphonies. For "the musician [to have] a precise and complete idea of the symphony", she needs at least to consider a possible music score as a possible symphony. In other words, our definitions should enable us to discriminate acceptable symphonies from music scores without a musical relevance. A fair generic description of acceptable symphonies would require a generic understanding of how symphonies work in the sense of having a musical meaning where the various possibilities would be understood collectively. There are two issues in reaching such a generic understanding of symphonies.

First, musical meaning is not an intrinsic property of a sequence of musical signs. Instead, musical meaning takes place in a historical, cultural context. For example, Erik Satie's or Moondog's work would probably not have made much sense for Bach. As a result, musical meaning is not just a function of the music score but also depends on the cultural context. Even though computers can transform music scores into sound automatically, a human interpret needs to be able to make sense of the music score. The situation is extremely similar to the reading out loud of a text which is very different when the text makes sense to the reader and when it does not.

Second, musical meaning depends on the specific arrangement of a musical piece and its many interwoven patterns (Mazzola, 2012). There are different qualitative patterns in music scores that may or may not make musical sense for readers. These patterns and their possible recurrences are specific properties of an individual symphony. The precise idea of a symphony includes these patterns and the meaning that they may evoke.

\subsection{Novelty in chaotic dynamical systems}

In this section, we will consider a dynamical system which should help to understand why the concepts of generic versus specific properties are necessary to avoid misconceptions about the concept of possibility. Let us consider an initial condition $0 \leq x<10$ and its decimal expansion $x=x_{0} \cdot x_{1} x_{2} x_{3} \ldots$. Then, we can define a dynamical system $u_{0}=x=x_{0} \cdot x_{1} x_{2} x_{3} \ldots, u_{1}=x_{1} \cdot x_{2} x_{3} \ldots$, $u_{2}=x_{2} \cdot x_{3} \ldots, \ldots$.

This dynamic is chaotic in the sense that more and more precise aspects of the initial conditions dominate the trajectory. For example, the integer part of $u_{10}$ is the $10^{\text {th }}$ decimal of the initial condition. Now, instead of using the decimal expansion, it is possible to use base 2 to obtain a binary representation of $x$ or instead to use base 27 and letters (and space) as symbols for digits. Let us do the latter and use the initial condition $x=$ w.hen a musician writes a symphony ... (with the rest of the initial condition following our translated quotation of Bergson). Then the integer part of $u_{n}$ spans the text of Bergson.

Does the dynamics $u_{n}$ tell us something about Bergson's text? Yes, in the sense that the text is a sequence of letters. However, this is valid for any text. Actually, another initial condition would have generated one of Shakespeare's play. Sadly, most of the initial conditions, that is to say, most real numbers, lead to texts that are meaningless for humans.

Chaotic dynamical systems may have rich dynamics, but, in a precise sense, they are not creating something new. Mathematically, the richness of their patterns stems from the fact that they are digging deeper and deeper into their initial conditions. Their analysis focuses on the way in which the dynamics are transforming the initial conditions, not the specific pattern stemming from a given initial condition.

It is not a generic property of the dynamics $u_{n}$ to generate a meaningful text. The mathematical analysis of the generic properties of $u_{n}$ does not involve the meaning of Bergson's text. In other terms, the odds to find initial conditions of $u_{n}$ that generate Bergson's text are almost null. It is necessary to have written this text beforehand to choose initial conditions leading $u_{n}$ to generate it.

A similar issue appears when Dawkins designs a toy computational model of evolution to show that variation and selection can lead to a specific result: the string $A$. In this model, a population of strings evolves by random variations and selection and converges towards $A$. Fitness is defined by the proximity to $A$. Then, $A$ is specified before the dynamics and cannot genuinely be said to emerge from it. Dawkins fully acknowledges this limitation: "phrases were judged according to the criterion of resemblance to a distant ideal target [...]. Life isn't like that" (Dawkins, 1986, p. 60). This kind of problems does not disappear easily. For example, (Adams et al., 2017) perform very interesting simulations of dynamical 
systems to study the appearance of innovations. However, when they allow dynamical rules to change, they change among predefined possibilities.

\subsection{Different notions of possibilities}

We propose to depart sharply from a naive set-theoretic view of possibilities, where the definition of a set would define each of its elements as possibilities and each of its subsets would be valid sets of possibilities. Instead, we advocate a theoretical notion of possibilities, which we also call explicit possibilities, where possibilities are defined if and only if we define explicitly also how they "work", that is to say how they take place in an appropriate theoretical framework where they have meaning. Our notion of possibility is based on what the theoretician can effectively express with her definitions.

Now, we can explain the conceptual articulation between the set of possible music scores $M_{s}$ and the possible symphonies. The set of possible music scores has generic properties that are relevant for writing, writing software or printing. These operations are its natural theoretical context. Music scores are used to communicate symphonies as a writing system, which means that they are typically sufficient for constraining the receiver for her to interpret some music scores as symphonies. However, the theoretical construct used to define $M_{s}$ is not sufficient for a sound theoretical understanding of symphonies themselves. This limitation is not just due to $M_{s}$ lacking generic constructs. Instead, the meaning of musical possibilities lies at the individual level which means that musical sense is not a generic property. Thus, $M_{s}$ may only be seen as a set of pre-possibilities for symphonies and not as a set of explicit possibilities.

We call "pre-possibilities" sets whose meaning is not entirely explicit for the intended phenomena. Pre-possibilities are usually possibilities defined in an initial theoretical contex $\sqrt{6}$ that are used in another context where they do not meet the criteria of explicit possibilities.

Elements of a set may have the status of pre-possibility for epistemic or objective reasons. Epistemic reasons correspond to a lack of knowledge, typically when the generic definition of pre-possibilities can be completed with other generic constructs that endow them with a satisfying theoretical structure for the intended phenomena. For example, a set of pre-possibilities can be endowed with probability distributions. However, the status of pre-possibilities can also have a more objective nature. When specific properties of individual elements are what matters, then we cannot transform a generic set of prepossibilities into explicit possibilities. Then, only some pre-possibilities can be completed to be explicit possibilities. In this case, the notion of new possibilities is irreducible at the theoretical level.

\footnotetext{
${ }^{6}$ Indeed, a definition is needed to talk about a set of pre-possibilities. As a result, pre-possibilities are defined for some operations.
}

In this second case, a set of pre-possibilities is fragile theoretically since this set is not defined by an adequate theoretical structure: it is irreducibly between two frameworks. For example, music scores are fundamentally between music and the concrete activity of writing. $\mathrm{Mu}$ sic scores are a limit case since they seem sufficient to represent symphonies and to communicate them. Let us illustrate the theoretical fragility of this set.

The relation between music scores and symphonies is not as simple as an automatic mapping. Between the music score and the symphonies played by an orchestra, there are interpretations and musical phrasing which lead to many versions corresponding to the same music score. Moreover, if one defines generic music scores strictly, then musical works will overflow this definition. For example, frequent changes of time signatures were foreign to classical music. Musical notations themselves are adapted to different styles and may be seen as openended. A musical notation is not a fundamental invariant of music. Thus, the notations do not define musical possibilities and composing symphonies is not an exploration of the space of music scores. Instead, musical notations enabled the practice of classical music and reciprocally are determined by the historical changes in the practice of music.

\subsection{Conclusion on the paradox}

The core of Bergson's argument is the identity of having a clear idea of a possible symphony and actually composing it. If Alice thinks about the possibility of a symphony and has an exhaustive account of this possible symphony, this symphony exists and Alice is its author. The issue that we have raised is that the set of possible music scores $M_{s}$ is mathematically well-defined and thus is a candidate for stating that all symphonies are defined as possibilities before they are conceived. However, the generic definition of $M_{s}$ is not equivalent to defining possible symphonies ahead of conceiving them because criteria to make musical sense explicit are not embedded in this description. They are not embedded because musicality is not attached to generic, collective properties of the elements of $M_{s}$. Instead, musical meaning corresponds to specific, individual properties of some elements of $M_{s}$. Thus, we ultimately agree with Bergson: the possibility of a symphony does not preexist this symphony.

\section{Novelty and possibility spaces in biology}

We will now apply our concepts to biology. In a first section, we discuss a few examples of sets that are sometimes considered as possibility spaces when they should be considered as pre-possibilities. Then, we discuss another approach to novelty that stems from biophysical models. This notion is weaker than the notion of new possibilities but leads us to useful considerations proper 
to natural sciences. We finally elaborate on specificities of biological novelties and discuss possible objections.

\subsection{Application to biology}

Some biologists and physicists consider that, in biology, mathematical spaces play a similar role than the mathematical spaces of physics. We will study several cases and argue that their role is closer to the one that music scores play for symphonies.

\subsubsection{The space of possible DNA sequences}

As a first example, let us consider complete genetic determinism, that is to say, the assumption that DNA sequences entirely determine phenotypes. This viewpoint is no longer dominant since the roles of the environment (Gilbert \& Epel, 2009) and of random factors (Paldi, 2003; Heams, 2014; Montévil et al., 2016) are increasingly becoming acknowledged. Nevertheless, it is interesting to confront our notion of possibility with this frame since its determinism seems incompatible with a strong notion of novelty.

The space of possible DNA sequences, $D_{s}$, is the set of finite sequences of the four symbols $A, T, G, C$. Under the assumption of genetic determinism, $D_{s}$ is sufficient to determine phenotypes. In the line of our former discussion, there are several possibilities for the relation between DNA sequences and phenotypes.

i) This relation is generic and is conceptually similar to the situation in statistical mechanics where generic properties of microstates are causally relevant. In this context, it is fair to say that all possibilities are predefined.

ii) The relation between DNA sequences and phenotypes is similar to the relation between music scores and symphonies. It depends on individual sequences. Let us recall that the space of music scores is appropriate for music writing software or printing. Similarly, the generic properties of the space of DNA sequences would be appropriate to understand methods like sequencing or phenomena like DNA replication. However, it would not be sufficient to state explicitly possible phenotypes. As such, $D_{s}$ would be a set of pre-possibilities.

The relation between DNA and phenotypes has never been described explicitly by a generic causal structure. The genetic code is a partial bridge between the two. However, this generic relation between mRNA sequences and amino acids sequences is not sufficient to determine the proteome or even proteins. The relation between individual sequences and protein shapes has a complex structure (Stadler et al., 2001). Moreover, determinants of this relation include alternative splicing, epigenetic effects, non-coding RNA and the proteome dynamics itself which all push the relation between DNA sequences and the proteome away from a straightforward application of the genetic code (David et al., 2013; Huang, 2009). These phenomena tend to make gene expression contextual and lead to consider that inheritance is the locus of a coupling between physiology and evolution (Danchin \& Pocheville, 2014). At the evolutionary level, there is a fundamental reason for the lack of a generic relation between DNA and phenotypes: this relation is not a theoretical invariant and, a priori, nothing prevents it from changing in evolution - except when changes lead to non-viable variants. Current life forms include diverse accumulations of such changes.

In conclusion, the space of DNA sequences $D_{s}$ is a theoretical construct that is not sufficient to discuss the phenotypes, starting with their viability. Then, the status of $D_{s}$ w.r. to phenotypes is similar to the status of the space of possible music scores $M_{s}$ w.r. to symphonies. The latter is not sufficient to assess whether music scores are symphonies or not. The functioning of organisms is not a generic property that can be discussed on the basis of the possible DNA sequences alone; it includes specificities proper to different phyla and even proper to some individuals. $D_{s}$ defines only pre-possibilities for phenotypes. As a result, even in the framework of complete genetic determinism, it seems necessary to consider that new possibilities appear in evolution..$^{7}$

\subsubsection{Networks and shapes}

The same reasoning applies to other mathematical spaces used to describe living phenomena, such as networks of chemical interactions or spaces of possible biological forms.

An important extension of molecular biology discusses networks of interacting molecules, where interactions are of a chemical nature. This extension defines the field of molecular systems biology. However, biological organizations do not correspond to generic properties of these spaces (possible networks endowed with one structure or another). Network structures are not exhaustive since relevant properties are excluded such as anatomical structures or physical forces. Remarkable evolutionary novelties at the molecular level such as molecular motors (Chowdhury, 2013), microtubules (Karsenti, 2008), chromatin (Cortini et al., 2016) or fibers (Barnes et al., 2014) are excluded from the discussion because their causal role does not correspond to generic chemical reactions. All these molecules are examples of molecules with specific properties that appeared in evolution. Chemical networks with particular properties such as autocatalytic sets aim to capture a fundamental property of cells (Hordijk \& Steel, 2017) but the study

\footnotetext{
${ }^{7}$ Here, genetic determinism is postulated without an explicit generic causal rule linking DNA to phenotypes. This epistemological status is to be contrasted with the deterministic frame of classical mechanics where determinism follows from the generic application of the Cauchy-Lipschitz theorem on the equations provided by the fundamental principle of dynamics, see section 3.1 .3
} 
of their generic properties does not capture the specific properties emerging in evolution.

Similarly, physicists interested in biological morphogenesis might want to consider the mathematical space of possible forms in the usual three-dimensional space or the possible positions of a finite number of cells in space. These shape spaces might seem all-encompassing in that they are typically used to describe biological shapes like music scores are used to describe symphonies. However, they are insufficient to describe many models of morphogenesis which typically involve chemicals (morphogens), fibers, mechanical forces, etc. These elements are required to make any biologically meaningful analysis in these spaces and are the historical outcomes of evolution. As a result, relevant properties are not generic features of these spaces which we relate to the critic of D'Arcy Thompson by Gould (2002, chap. 11). These spaces are interesting as pre-possibilities, like music scores for symphonies, but they are not appropriate to prestate $e x$ plicitly all possible organisms.

\subsubsection{Biological possibilities and classical mechanics}

We now discuss a more philosophical way to criticize the notion of new possibilities and diachronic emergence. The idea is to propose a putative definitive space of possibilities by relying on physics, usually classical mechanics, on the basis of a physicalist and reductionist view of biology. In classical mechanics, a system such as an organism or the biosphere should follow a specific trajectory that follows from its state at a given time point, where the state is defined as the positions and momenta of the particles involved. We will call $S$ the space generated by these quantities. Note that this reasoning is not entirely sound from a physical point of view but it is common and interesting: $!^{8}$

Let us now analyze this situation precisely. The fundamental principle of classical mechanics states that, for each particle, mass times acceleration equals the external forces exerted on this particle. Therefore, we have a huge dynamical system $\phi$ which is written on the basis of generic forces. Determinism follows from the application of the Cauchy-Lipschitz theorem which ensures that this kind of dynamical system has a unique solution for a given state at a time $t_{0}$. In short, determinism is a generic property of such dynamical systems. There are a few other generic properties of these systems such as the conservation of energy, of momentum, Etc.

However, it does not follow that this generic construct would explicitly define the possibilities of biological evolution or biological organisms. Actually, the

\footnotetext{
${ }^{8}$ Organisms are open systems, with fluxes of matter and energy that are not straightforward to describe in classical mechanics and pertain more to far from equilibrium thermodynamics. With the natural history in mind, the only relevant isolated system would be the solar system. Moreover, biology also involves chemical reactions which, in physics, pertain to quantum mechanics and not classical mechanics.
}

sets involved have the same cardinality as real numbers, and this cardinality implies that we cannot define individually all their elements, as discussed in section 2.1 . Then, it is not sound to claim that biological possibilities can be derived from physical ones without a very precise discussion.

Nevertheless, we cannot conclude immediately that this conclusion is wrong. We have to discuss whether individual properties of the elements of the state space $S$ are theoretically relevant for biology. Since the system is deterministic, these individual properties ultimately correspond to the properties of the initial conditions w.r. to $\phi$. The question is then to assess whether generic properties of $\phi$ are sufficient to understand biological possibilities, or on the opposite, if biology is mostly about specific properties of the initial conditions of $\phi$. In this deterministic frame, all contingent events come down to specific properties of initial conditions. Therefore, all arguments which state that such events are decisive for biological phenomena (for example, Beatty, 1995, Gould, 2002; Montévil et al., 2016) can be translated into arguments to state that biology depends on specific properties of these initial conditions. As a result, we do not think that this system defines explicitly biological possibilities. We provide further arguments in this sense in section 3.3 .

We have examined a few examples of putative allencompassing sets and shown that they are compatible with our notion of new possibility. Like the situation in music and unlike the one in statistical mechanics, these spaces do not provide an explicit account of biological possibilities.

\subsection{Novelty and physical approach to self-organization}

To understand development, several biologists and physicists use the concepts of phase transitions, physical morphogenesis or the associated concept of physical self-organization (Moore, 2012; Zhu et al., 2010; Saetzler et al., 2011; Forgacs \& Newman, 2005). Turing's model of morphogenesis (Turing, 1952) typically falls in this category. Other examples are phase transitions such as the formation of graphite mentioned in the introduction, Bénard cells or flames. The corresponding models focus on the formation of a qualitative "new" structure. These concepts have different theoretical backgrounds (Anderson \& Stein, 1985) but their mathematical approaches to novelty are sufficiently close for us to discuss them together.

The novelty described by these models constitutes a paradigmatic case of diachronic emergence and typically corresponds to a symmetry breaking (Anderson, 1972; Longo \& Montévil, 2014). In a nutshell, symmetries are transformations which do not change an intended aspect of an object. This concept applies both to the usual three-dimensional space and more abstract spaces. In a symmetry breaking, the whole description of the object, the state and the equations, initially follows a symmetry. 
For example, the system may be symmetric for all rotations about a point, which means that all directions are equivalent. After the symmetry breaking, one or several directions are no longer equivalent to the others. Since both the initial state and equations are symmetric, the "choice" of specific directions does not derive from the initial description and is random $\sqrt{9}^{9}$ Moreover, these new directions are associated with specific properties and thus correspond to a new qualitative behavior. As a result, in these frameworks, novelty stems from the randomness associated with the symmetry breaking. Nevertheless, several related reasons restrict the strength of this concept of novelty.

First, these phenomena are spontaneous and may be repeated ad libidum. In models, physical self-organization is generic. It is usually sufficient to change the value of a parameter for the new structure or dynamics to appear. For example, it is sufficient to lower the temperature of liquid water to trigger the formation of ice. There is randomness in these phenomena but this randomness only corresponds to the way in which the symmetry is broken, or in other words the "choice" of one direction or another. The qualitative aspect of the pattern are always the same (and actually all possible outcomes are equivalent). As a result, contingency and a fortiori unpredictability do not impact the qualitative outcome. This property weakens the notion of novelty associated with these processes (Stephan, 1999).

Second, physicists mathematize these phenomena on the basis of invariance. Equations are crucial to understand these systems and these equations do not change during the formation of new structures, at least at the microscopic level. For example, dynamical systems follow the same rules during the dynamics. Similarly, the same equation describes the partition function before and after a phase transition. At the same time, the macroscopic level includes a variable called the order parameter which goes from being uniformly zero to having a non-trivial value. Thus, we can say that the macroscopic equation changes. Statistical mechanics is intrinsically ambivalent since the microscopic equation does not change while the macroscopic one does: there is a duality between the two levels. Nevertheless, this means that, at the microscopic level, the causal structure remains the same before and after the change. As a result, the new patterns stem from a preexisting mathematical structure. The spontaneous nature of these novelties follows from the permanence of these underlying equations, and this permanence justifies that the changes are generic: once a parameter reaches a value given by the equations, the novelty has to appear.

\footnotetext{
${ }^{9}$ This line of reasoning is very general, but the corresponding randomness can be interpreted in different ways depending on the theoretical context. In classical mechanics and related deterministic frameworks, this randomness stems from measurement: the state of a system cannot be determined empirically with infinite precision which entails unpredictability, see Longo \& Montévil (2017) for a general discussion.
}

Third, the permanence of the equations corresponds to the permanence of a causal structure. For example, a phase transition is the transition of local fluctuations to system-wide effects. The novelty follows from a causal structure that is already relevant and already actual. We propose then to distinguish virtual possibilities from actual possibilities. Virtual possibilities do not follow from the causal relations required to understand the initial situation. For example, Adams et al. (2017) uses virtual possibilities by writing a dynamical system which switches between unrelated rules. By contrast, actual possibilities are possibilities which may be qualitatively different but are nevertheless entailed by the relations between the parts of a system before the possibility becomes actualized. In physical models, the novelty is not just virtual in the initial situation; its formal ingredients are already there. Therefore, the new patterns were actual possibilities before their appearance.

Fourth, in these frameworks, the formation of a new structure is generally punctual: below a given value of the control parameter, the new structure does not appear, and it does appear above this value. The only middle ground corresponds to a point. Examples include phase transitions or bifurcation points for dynamical systems. It is simple to understand this when the formation of the structure corresponds to a symmetry breaking. Let us recall that a symmetry breaking is a transition from a symmetric situation to a situation with fewer symmetries such as the transition from being symmetric by rotation to having special directions. Since having a given set of symmetries is a property that is either met or not, the transition from the first to the second is an all or nothing phenomenon. Because these transitions are all or nothing, they cannot be decomposed and thus are elementary. Being elementary, they are easier to trigger than more complex novelties. In short, the punctual nature of the appearance of these new structures corresponds to their elementary nature and contributes to explaining why such changes occur spontaneously.

Fifth, in these models, the set of qualitatively different macroscopic patterns is usually very small. The examples in the beginning of this discussion lead to a finite and actually a small number of such possibilities which means that they can all be predicted, provided that the equations describing them remain valid. By contrast, following our discussion in section 2, the notion of new possibilities becomes irreducible when all relevant qualitative cases cannot be analyzed.

Last, these systems are mostly ahistorical, and this is crucial for the concept of novelty. It does not matter whether a volume of liquid water used to be in a solid state in the past or if it is the first time that this specific volume of water transforms into ice. The transformation from liquid to ice is the same independently of whether the past of the system includes this state or not. In this sense, the exploration of macroscopic possibilities has no permanent consequences for the system beyond the 
permanence of the realization of these possibilities. By contrast, novelties in biology can have consequences that are not limited to their preservation. The appearance of feathers in some dinosaurs has led to changes in the organizations of these dinosaurs over evolutionary time scales which means that the impact of this appearance is not limited to the permanence of the novelty. Similarly, the specific way in which a two-legged goat learned to walk has led to anatomical accommodations which facilitate this behavior (West-Eberhard, 2003). Biological novelties typically lead to changes beyond just their appearance.

Let us conclude on the concept of novelty in these frameworks. We do not aim to criticize the idea that these models correspond to a genuine and objective notion of novelty. However, this notion is a weak one for all the reasons above. In particular, it is insufficient to justify and understand the concept of new possibilities or the historical nature of biological phenomena. These physical novelties correspond to elementary, punctual and generic processes.

\subsection{What matters for organisms}

\subsubsection{The importance of functions}

In the previous discussion, we have left implicit what matters in the causal structure of organisms. We think that the proper understanding of organisms or species has to include the many functions that contribute to their organization, survival, and reproduction. As a result, biology has a special interest in parts that are functional and in what they do. This reasoning is at least partially in line with Mayr's statement quoted in introduction (Mayr, 1963). It follows that generic spaces which do not articulate these aspects cannot provide an explicit account of biological possibilities. They can play an explanatory role, but only as pre-possibilities.

Our perspective, here, differs from phylogeny where structures and more precisely synapomorphies (shared novelties) are used to classify organisms. This methodology has been chosen because phylogeny aims to assess genealogical relationships. In our terminology, the best properties for phylogeny are the ones that are sufficiently specific to be unlikely to appear several times in evolution and at the same time generic and stable enough to be shared by genealogically close individuals, up to possible variations. Concerns in discussing functions stem from the idea that similar functions can mold structures towards the same optimal shape so that genericity could be obtained without common descent. However, we can point out that the same applies to elementary morphogenetic processes. For example, Thom's catastrophe theory provides a systematic, ahistorical classification of at least some of these processes. In the case of morphogenesis, Wagner \& Lynch (2010) uses gene networks called character identity networks precisely to ensure the specificity of the novelties. Ultimately what matters is the specificity of the novelty in combination with its theoretical relevance.

We will now provide a further justification of the importance of biological functions. As a thought experiment, let us consider entirely silent point mutations that are subject to drift, that is to say, fixation for purely statistical reasons. Assuming there is no lateral transfer and that the sequences are very long, the proximity of two sequences have vanishingly small chances to be obtained without genealogical proximity. This is due to the huge number of possible sequences which prevents ergodicity in practice, that is, the exploration of the full possibility space, see Longo et al. (2012). The uniqueness of the outcome is useful to reconstruct genealogies, but it does not mean that the theoretically relevant causal structure is specific. On the opposite, this situation is perfectly well described by the generic process of drift and equiprobable mutations. The causal analysis of the situation is provided by the generic analysis of such a process. By contrast, if there is a feedback between the specificity of a situation and the causal analysis, then there is a strong historicity that prevents the specific situation to be subsumed by a generic analysis. In biology, we then posit that historicity stems from the coupling between specificity and functionality. In the following discussion, we will focus on novelties which are associated with biological functions.

Biological functions may be interpreted in different ways depending on the level of description and the theoretical perspective of interest. We discuss two main philosophical accounts of biological functions, and we consider that they are not mutually exclusive. The first framework is called the selective effect account of functions. In this account, heritable traits are functional when, in a nutshell, their consequences have led them to be positively selected (Godfrey-Smith, 1994; Millikan, 1989; Neander, 1991; Garson, 2016). The second framework is called organizational and states that functionality stems from being included in the circular causal structure that characterizes organisms (Mossio et al., 2009, Montévil \& Mossio, 2015). This perspective is in line with former works of Varela et al. (1974), Rosen (1991) and Kauffman (2002). In the framework of closure of constraints, functional parts are called functional "constraints". A constraint is defined by its causal role with respect to a process and its stability at the time scale of this process: it is not consumed nor destroyed by the process. Let us call $\mathscr{C}$ the set of constraints that are part of an organism. For a constraint $c$ to be in $\mathscr{E}, c$ needs to act on at least a process generating another element of $\mathscr{C}$ and to depend on at least another element of $\mathscr{C}$. In a nutshell, constraints of an organism are collectively mutually dependent (Montévil \& Mossio, 2015; Mossio et al., 2016). Since the existence of these constraints depends on their consequences via the other constraints of $\mathscr{C}$, it is relevant to interpret them as being functional.

Before elaborating on the consequences of these frame- 
works on novelties, let us consider the reciprocal question and remark that novelties make it possible to discuss differences between the selective effect and the organizational accounts of functions. Novelties that are restricted to an individual such as the two-legged goat mentioned in section 3.2 cannot be etiological functions since they are not heritable. However, they certainly can be functional in the organizational sense. This example implies that the organizational notion cannot be reduced to the etiological one.

\subsubsection{Novelties and functions}

In both accounts of functions, relevant biological novelties are not just the appearance of patterns. In the selective effect account, defining a function requires discussing its differential effect on the life cycle in a population. In the organizational account, the concept of function directly involves the relationship between the part of interest, associated parts, and ultimately the rest of the organism. In both accounts, the relationships between the part studied and a larger whole are fundamental, and this applies to biological novelties inasmuch as we assume that they are functional.

As mentioned in the previous section, novelties in the sense of a single symmetry breaking are limited by the fact that they have no lasting impact beyond the maintaining of the novelty itself (that is the maintaining of the symmetry breaking). It is not the case for functional novelties. In the organizational account, biologically relevant novelties are constraints that become a part of the organization. By definition of an organization, a relevant novelty i) contributes to the maintaining of at least another constraint of the organization and ii) is maintained by processes which are canalized by at least another constraint of the organization. Then, by contrast with the physical novelties in the previous section, the appearance of a biological novelty is generally not punctual. The relations leading to i) and ii) do not necessarily appear simultaneously. The appearance of a biological novelty is then a composite event that corresponds to the integration of the novelty to an organization. In this framework, the focus on functions does not mean that just the role of a part is considered. Instead, it means that both criteria i) and ii) are met. The constraint plays a role and this role is performed in a specific manner by generating other constraints. However, this is not sufficient, and it is also necessary to make explicit its dependence on other constraints. The taking into account of these relations means that the organizational framework reduces the gap between purely functional and structural perspectives on novelties. Moreover, the integration of a constraint to an organization is not restricted to the minimum requirements i) and ii). Instead, this integration may become more intricate over time by the articulation with various other, possibly new constraints.

The point is that the theoretical description of functions is no longer elementary in relational accounts such as closure of constraints. For a functional constraint to be an explicit possibility, it is required to define it and to show that i) and ii) are met and lead the constraint to be a part of closure. We illustrate this by discussing a few cases.

- Let us consider a part that can be described with the (bio)physical concepts and models discussed in the previous section. Then, changing the value of a parameter leads to a new constraint. At the level of the part, the constraint appears as the generic result of a causal structure that is already actual, it corresponds to an actual possibility. However, these models are not self-sufficient since the inscription of the new constraint in organizations is not made explicit. More precisely, condition ii) can be met "for free" in some cases since the constraints described by the models are already actual and are presumably maintained by other constraints.

Now, if the relation between the new constraint and the organism can be deduced from the current state of affairs, then this new constraint was an actual biological possibility before it appeared.

If not, the new constraint is an actual biological pre-possibility. In this case, the novelty does not stem just from the new constraint per se but instead from its inscription in the organism. Its possible role, i), is not predefined.

- Let us assume that a functional role is associated with a part. Then, this role may enable us to define a specific pre-possibility as biologically relevant. Several biophysical models uses this argument (for example, Lesne \& Victor, 2006; West et al., 1999). For example, the specific situation may be optimal w.r. to this role or have functionally remarkable qualitative features. Then the new constraint appears as a specific pre-possibility in the description of the part, but it can be seen as a generic outcome when taking into account its functionality. In this case, it is condition i) which tends to be met directly. But it is not always the case since the specific situation may require a reorganization of the way the role of the functional part is performed. Moreover, condition ii) is not met a priori since this situation is not generic in the description of the part and thus requires an explanation. For example, being at a bifurcation point requires the addition of an entirely different regulatory dynamics (Camalet et al., 2000).

- Now let us consider one or several new constraints that were not actual possibilities before they appear. In this situation, the novelty is a virtual biological possibility if its articulation is also defined and a virtual biological pre-possibility if it is not. 
For example, Adams et al. (2017) define a computational dynamics with changes of dynamical rules along the dynamics. The mathematical structure of these rules cannot be deduced from each other. As a result, when one rule is actual the alternative is virtual: its possibility is not deducible from the current state of affairs. If the new constraint is elementary, it corresponds to a next adjacent possible in Kauffman's vocabulary (Kauffman, 1996; Longo et al., 2012).

In the two first cases, the new constraint itself can be defined as a generic outcome, either as a result of a morphogenetic process or for functional reasons. However, this is not usually sufficient to describe the new constraint as a possible part of the organization since its articulation with the organization is not made explicit. This articulation may even be impossible or require many other changes. The generic definitions of the constraints do not show explicitly that these generic constraints can be articulated to the organization and how. As a result, they are only pre-possibilities. Then, the appearance of the new functional constraint corresponds to a new possibility.

In the last case, the new constraint is neither an actual pre-possibility at the level of the part nor generic from a purely functional point of view. This constraint is not deduced from the causal structure of the initial organization. As a result, its description must have another origin. In the example considered (Adams et al., 2017), the new rules were postulated as a way to perform simulations. In a more biological perspective, it is possible to define virtual possibilities by analogy with other phyla. In any cases, we consider that virtual possibilities are not genuine possibilities because they are not actual possibilities: they do not stem from the relations needed to understand the current state of affairs. Should they get actualized, then they are new possibilities.

At this point, we have not argued whether the status of pre-possibility is objective or epistemic as defined in section 2.4. In general, we define possibilities as actual, generic possibilities in the initial situation at the level of organizations. The latter implies that they meet conditions i) and ii). This objective, positive notion of possibility allows distinguishing artificial constructs such as virtual possibilities from genuine possibilities.

We will now argue that there are objective new biological possibilities. We mentioned in the preceding section, point three, that physical models of morphogenesis are based on a causal structure that is already actual and not merely virtual. In biology, this perspective is not valid in general. For example, let us consider two novelties, where the second novelty requires the first novelty not only to appear but also to acquire a biological meaning. In other words, the emergence of the first novelty is a necessary ingredient for the second novelty to be able to play a functional role. Then, the causal structure of the second novelty is clearly not involved in the causal structure of the initial situation. For example, articulated jaws enabled teeth such as molars which can crush food. However, crushing food with the mouth was and still is not an actual possibility at all for Chordates without articulated jaws.

This difference between physical morphogenesis and biology should not come as a surprise, even from a reductionist point of view. Physical morphogenesis is a framework for systems which are made from predefined components and boundary conditions and aim to derive the appearance of a new structure from these already existing interactions. Biological changes are not bound by these limitations.

A part may be involved in the appearance of other new possibilities, and this form of causation has been called enablement (Longo et al., 2012; Longo \& Montévil, 2013b). One of the core processes of evolution is the iterative appearance of novelties on the basis of already existing organizations. This iterative process is central to the open-endedness of biological evolution. Then, a novelty may become deeply integrated into certain biological organizations, making its complete disappearance unlikely to be viable. For example, thyroid hormones appeared and are shared among vertebrates. Some of their effects are largely conserved (Tohme et al., 2012) but others are highly specific such as their role in many specific metamorphosis processes (Holzer \& Laudet, 2015).

To sum up, biological novelties are not elementary events. Instead, they involve the integration to an organization, a life cycle and an environment and this integration typically involves a sequence of changes. It justifies that biological novelties are specific even when some aspects of them are generic. As a result, biological changes involve non-generic changes, and we think that the concept of new possibilities is fundamental for biology.

\subsection{Responses to possible mathematical objections}

A possible mathematical argument against the notion of new possibilities in biology is based on dynamical systems. Some dynamics are indeed very rich in the sense that they can generate many patterns. For example, the dynamical system described in section 2.3 can generate all possible strings of characters. However, as discussed in the same section, it is not sufficient for a dynamics to be able to generate a pattern of interest for this dynamics to actually explain this pattern, that is to say for this pattern to be an explicit possibility of this system. If this pattern stems from a specific initial condition, then this pattern is actually more a property of the specific initial condition than of the rule of the dynamics per se. Therefore, it is necessary (from a statistical or metric point of view) to artificially choose the initial condition that leads to this pattern for the pattern to actually appear. 
Another mathematical counterargument states that we can propose abstract spaces so large that they can accommodate everything that biologists can encounter (for example a space of infinite dimension). This argument differs from the discussion in section 3.1 since this mathematical space is explicitly built without an interpretation (unlike the spatial position of cells, for example). The response remains similar; these spaces are not endowed with mathematical structures such as equations that would make their biological meaning explicit. As such, these spaces do not enable scientists to state biological possibilities, and do not oppose the notion of new possibilities.

Last, a possible objection is based on the following operation: after the observation of a new possibility, this new possibility can be added to the initial set of possibilities. There are two possible relations between a set of pre-possibilities $S$ and a situation $a$ which does not correspond to generic properties of $S$. First, $a$ may be an element of $S$. Second, $a$ may be outside $S$. Going from one of these two situations to the other is to a certain extent arbitrary because $S$ can be extended $a$ posteriori by adding new possibilities. However, in both cases, the properties of $a$ remain non-generic in the initial description which means that there is objectivity in describing $a$ as a new possibility even if we accept this retrospective theoretical move.

Now, a further counter-argument would be to change the definition of $S$ a posteriori so that the new possibility $a$ becomes generic. This objection requires a precise discussion. Let us call $S(t)$ the possibility space at time $t$. If the observer witnesses a new possibility at time $t^{\prime}>t$, then the possibility space $S\left(t^{\prime}\right)$ is larger than $S(t)$. The operation that we have described in the previous paragraph is retrospectively to consider $S_{t^{\prime}}(t)$, the space of possibility at time $t$ on the basis of a novelty that appeared between $t$ and $t^{\prime}$. Bergson calls conflating $S_{t^{\prime}}(t)$ and $S(t)$ the retrospective illusion. This illusion may be compared with the situation in usual probabilities. Using the result of a random drawing to describe the initial condition makes it always possible to describe the process as deterministic which is clearly wrong at this level of description. The novelty used to define $S_{t^{\prime}}(t)$ by comparison with $S(t)$ does not come from the actual behavior at $t$ or before, ex hypothesi. The cost of conflating $S_{t^{\prime}}(t)$ and $S(t)$ is that the definition of $S_{t}$ depends on ulterior phenomena and becomes a finalist description: this methodology has a bias towards a specific outcome by excluding many alternative changes which are not taken into account.

\section{Conclusion}

In this paper, we discuss the concept of novelty in music and biology, and we justify the notion of new possibilities. Our argument starts with a paradox stemming from the analysis of Bergson's work. For Bergson, the possibility of a symphony does not preexist to its conception because knowing the possibility of the symphony implies that the symphony exists. However, we point out that the set of possible music scores is mathematically well-defined and this set seems to define all possible symphonies. The confrontation of this two lines of reasoning constitutes a paradox.

To solve this paradox, we have shown that defining a set is not the same as defining each of its elements individually. More generally, generic, collective definitions and reasoning cannot be conflated with reasoning on individual elements. In physical models and theories, generic properties of sets of possibilities are the theoretically relevant properties. As a result, physicists can discuss huge possibility spaces where the physical, causal properties of these possibilities are made explicit. By contrast, in music, an examination of individual music scores is ultimately necessary to discuss their musical meaning.

We then define explicit possibilities, which are endowed with an explicit discussion of the relevant properties. When sets are infinite, explicit possibilities require the genericity of the relevant properties, except for a finite number of specific cases. By contrast, prepossibilities are relevant sets which do not meet the criterion of possibilities. When the relevant properties are specific, the status of pre-possibility is not due to a lack of knowledge, and the notion of new possibility is objective.

In biology, some mathematical structures are often assumed to be sufficient to represent or even determine organisms. For example, complete genetic determinism assumes that DNA sequences are sufficient to determine phenotypes. We show that even this extreme assumption is compatible with the idea of new possibilities because such constructs define pre-possibilities and not possibilities. For example, there is no generic relation between genotypes and phenotypes. Instead, this relation changes in evolution. Organisms have specific features that are not covered by the generic properties of mathematical structures such as sequences of nucleotides. Then, the theoretical roles played by such spaces cannot be compared with the ones in physics, where the causal structure of the possibilities is made explicit.

We also discuss the idea that biological situations could be seen from the perspective of classical mechanics, with a fixed possibility space and dynamical rule. We show that there is no reason to think that biological properties are generic properties of such a system which means that biological explicit possibilities are not necessarily derived from the physical ones. This reasoning is based on the weight of historical contingency in the determination of biological processes and provides a strong argument for diachronic emergence in biology.

We analyze novelty in some physical models. We use these examples to distinguish virtual possibilities from actual possibilities: the latter are the result of a 
pre-existing causal structure that is already taking place. In these models, the concept of novelty is objective but weak since these novelties are generic, actual possibilities before they appear.

In biology, we argue that a strong notion of novelty is given by situations which are specific before being actualized and are associated with functions. Processes leading to specific outcomes are the ones which are likely to have a unique origin. However, they are not sufficient to argue that new possibilities are relevant. Drift in huge spaces provides a weak form of historicity that can be analyzed by generic equations. By contrast, as discussed in section 3.3.1, if there is a feedback between the specificity of a situation and the causal analysis, then there is a strong historicity that prevents the specific situation to be subsumed by a generic framework. From this perspective, we think that functional novelties have a special role. Then, we discuss the properties of biological novelties and show that they are composite. As a result, even in cases where partial generic predictions can be performed, functional novelties are typically specific. As a consequence, we think that the concept of new possibilities is a fundamental biological concept.

\section{Acknowledgements}

I am grateful to Ana Soto, Giuseppe Longo, Carlos Sonnenschein, Marc Godinot, Paul-Antoine Miquel, Arnaud Pocheville and the anonymous reviewers for their critical insights on previous versions of this article. I also would like to thank Guillaume Lecointre for helpful discussions and Jean Lassègue for pointing out the work of Leibniz.

\section{References}

Adams, A., Zenil, H., Davies, P., \& Walker, S. (2017). Formal definitions of unbounded evolution and innovation reveal universal mechanisms for open-ended evolution in dynamical systems. Scientific Reports, 7. doi 10.1038/s41598-017-00810-8.

Anderson, P. W. (1972). More is different. Science, 177, 393-396. doi $10.1126 /$ science.177.4047.393

Anderson, P. W., \& Stein, D. L. (1985). Broken symmetry, emergent properties, dissipative structures, life: Are they related? In E. F. Yates (Ed.), Self-organizing systems: The emergence of order (pp. 445458). Plenum Press.

Barnes, C., Speroni, L., Quinn, K., Montévil, M., Saetzler, K., BodeAnimashaun, G., McKerr, G., Georgakoudi, I., Downes, S., Sonnenschein, C., Howard, V., \& Soto, A. (2014). From single cells to tissues: Interactions between the matrix and human breast cells in real time. PLoS ONE, 9, e93325. doi 10.1371/journal.pone.0093325

Beatty, J. (1995). The evolutionary contingency thesis. Concepts, theories, and rationality in the biological sciences, (pp. 45-81).

Bedau, M. A., McCaskill, J. S., Packard, N. H., Rasmussen, S., Adami, C., Green, D. G., Ikegami, T., Kaneko, K., \& Ray, T. S. (2000). Open problems in artificial life. Artificial Life, 6, 363-376. doi 10. 1162/106454600300103683

Bergson, H. (2014). La pensée et le mouvant. Editions Flammarion.

Bich, L., \& Bocchi, G. (2012). Emergent processes as generation of discontinuities. In Methods, models, simulations and approaches towards a general theory of change. (pp. 135-146). Singapore: World Scientific.

Borges, J. L. (1998). The library of babel. Collected fictions, .
Camalet, S., Duke, T., Julicher, F., \& Prost, J. (2000). Auditory sensitivity provided by self-tuned critical oscillations of hair cells. Proceedings of the National Academy of Sciences, (pp. 3183-3188). doi $10.1073 /$ pnas.97.7.3183

Chowdhury, D. (2013). Stochastic mechano-chemical kinetics of molecular motors: a multidisciplinary enterprise from a physicist's perspective. Physics Reports, 529, 1-197. doi 10.1016/j . physrep.2013. 03.005

Cortini, R., Barbi, M., Caré, B. R., Lavelle, C., Lesne, A., Mozziconacci, J., \& Victor, J.-M. (2016). The physics of epigenetics. Reviewes of Modern Physics, 88, 025002. doi 10.1103/RevModPhys. 88 025002

Danchin, E., \& Pocheville, A. (2014). Inheritance is where physiology meets evolution. The fournal of Physiology, 592, 2307-2317. doi 10 1113/jphysiol.2014.272096

David, L., Ben-Harosh, Y., Stolovicki, E., Moore, L. S., Nguyen, M., Tamse, R., Dean, J., Mancera, E., Steinmetz, L. M., \& Braun, E. (2013). Multiple genomic changes associated with reorganization of gene regulation and adaptation in yeast. Molecular Biology and Evolution, 30, 1514-1526. doi $10.1093 / \mathrm{molbev} / \mathrm{mst} 071$

Dawkins, R. (1986). The blind watchmaker: Why the evidence of evolution reveals a universe without design. WW Norton \& Company.

Feigenbaum, M. J. (1980). The metric universal properties of period doubling bifurcations and the spectrum for a route to turbulence. Annals of the New York Academy of Sciences, 357, 330-336.

Forgacs, G., \& Newman, S. A. (2005). Biological physics of the developing embryo. Cambridge University Press.

Garson, J. (2016). A critical overview of biological functions. Springer.

Gilbert, S. F., \& Epel, D. (2009). Ecological developmental biology: integrating epigenetics, medicine, and evolution. Sinauer Associates Sunderland.

Godfrey-Smith, P. (1994). A modern history theory of functions. Noûs, 28, 344-362.

Gould, S. J. (2002). The structure of evolutionary theory. Harvard University Press.

Heams, T. (2014). Randomness in biology. Math. Structures in Comp. Sci., special issue, 24. doi $10.1017 / \mathrm{S} 096012951200076 \mathrm{X}$

Holzer, G., \& Laudet, V. (2015). Thyroid hormones: a triple-edged sword for life history transitions. Current Biology, 25, R344-R347. doi $10.1016 / \mathrm{j}$. cub. 2015.02.026

Hordijk, W., \& Steel, M. (2017). Chasing the tail: The emergence of autocatalytic networks. Biosystems, 152, $1-10$. doi $10.1016 / \mathrm{j}$ biosystems.2016.12.002

Huang, S. (2009). Non-genetic heterogeneity of cells in development: more than just noise. Development, $136,3853-3862$. doi $10.1242 /$ dev.035139.

Karsenti, E. (2008). Self-organization in cell biology: a brief history. Nature Reviews Molecular Cell Biology, 9, 255-262. doi 10.1038/ nrm2357.

Kauffman, S. (1996). At home in the universe: The search for the lawes of self-organization and complexity. Oxford university press.

Kauffman, S. (2002). Investigations. Oxford University Press, USA.

Kauffman, S. A. (2016). Humanity in a Creative Universe. Oxford University Press.

Koppl, R., Kauffman, S., Felin, T., \& Longo, G. (2015). Economics for a creative world. Fournal of Institutional Economics, 11, 1-31. doi $10.1017 /$ S1744137414000150

Koutroufinis, S. (2014). Beyond systems theoretical explanations of an organism's becoming: A process philosophical approach. In S. Koutroufinis (Ed.), In Life and Process (pp. 99-132). De Gruyter. doi $10.1515 / 9783110352597$

Koutroufinis, S. (2017). Organism, machine, process. towards a process ontology for organismic dynamics. Organisms. Fournal of Biological Sciences, 1, 23-44. URL:/http://ojs.uniroma1.it/index.php/ Organisms/article/view/13878

Leibniz, G. W. (1991). De l'horizon de la doctrine humaine. Vrin. 1666.

Lesne, A., \& Victor, J.-M. (2006). Chromatin fiber functional organization: Some plausible models. Eur Phys $\mathcal{F}$ E Soft Matter, 19, 279-290. doi $10.1140 / \mathrm{epje} / \mathrm{i} 2005-10050-6$.

Longo, G., \& Montévil, M. (2011). From physics to biology by extending criticality and symmetry breakings. Progress in Biophysics 
and Molecular Biology, 106, 340 - 347. doi $10.1016 / \mathrm{j} \cdot$ pbiomolbio 2011.03.005.

Longo, G., \& Montévil, M. (2013a). Extended criticality, phase spaces and enablement in biology. Chaos, Solitons \& Fractals, 55, $64-79$. doi $10.1016 / j$.chaos.2013.03.008

Longo, G., \& Montévil, M. (2013b). Extended criticality, phase spaces and enablement in biology. Chaos, Solitons \& Fractals, (pp. -). doi 10 $1016 / \mathrm{j}$.chaos.2013.03.008.

Longo, G., \& Montévil, M. (2014). Perspectives on Organisms: Biological time, symmetries and singularities. Lecture Notes in Morphogenesis. Dordrecht: Springer. doi 10.1007/978-3-642-35938-5

Longo, G., \& Montévil, M. (2017). Comparing symmetries in models and simulations. In M. Dorato, L. Magnani, \& T. Bertolotti (Eds.), Springer Handbook of Model-Based Science. Springer. doi:10.1007/ 978-3-319-30526-4

Longo, G., Montévil, M., \& Kauffman, S. (2012). No entailing laws, but enablement in the evolution of the biosphere. In Genetic and Evolutionary Computation Conference. GECCO'12 New York, NY, USA: ACM. doi 10.1145/2330784.2330946.

Loreto, V., Servedio, V. D. P., Strogatz, S. H., \& Tria, F. (2016). Dynamics on expanding spaces: Modeling the emergence of novelties. In M. Degli Esposti, E. G. Altmann, \& F. Pachet (Eds.), Creativity and Universality in Language (pp. 59-83). Cham: Springer. doi $10.1007 / 978-3-319-24403-7 \_5$

Mayr, E. (1963). Animal species and evolution volume 797. Belknap Press of Harvard University Press Cambridge, Massachusetts.

Mazzola, G. (2012). The topos of music: geometric logic of concepts, theory, and performance. Birkhäuser.

Millikan, R. G. (1989). In defense of proper functions. Philosophy of science, $56,288-302$.

Montévil, M., \& Mossio, M. (2015). Biological organisation as closure of constraints. Fournal of Theoretical Biology, 372, 179 - 191. doi 10 1016/j.jtbi.2015.02.029

Montévil, M., Mossio, M., Pocheville, A., \& Longo, G. (2016). Theoretical principles for biology: Variation. Progress in Biophysics and Molecular Biology, 122, 36 - 50. doi $10.1016 / \mathrm{j}$. pbiomolbio.2016.08.005

Moore, A. (2012). Life defined. BioEssays, 34, 253-254. doi:10.1002/ bies.201290011.

Mossio, M., Montévil, M., \& Longo, G. (2016). Theoretical principles for biology: Organization. Progress in Biophysics and Molecular Biology, 122, 24 - 35. doi 10.1016/j.pbiomolbio.2016.07.005

Mossio, M., Saborido, C., \& Moreno, A. (2009). An organizational account of biological functions. The British fournal for the Philosophy of Science, 60, 813-841. doi 10.1093/bjps/axp036

Muller, G. B., \& Wagner, G. P. (1991). Novelty in evolution: restructuring the concept. Annual Review of Ecology and Systematics, (pp. 229-256). doi 10.1146/annurev .es.22.110191.001305

Neander, K. (1991). Functions as selected effects: 'The conceptual analyst's defense. Philosophy of Science, 58, 168-184. doi:10.1086/ 289610

Pachet, F., \& Roy, P. (2014). Imitative leadsheet generation with user constraints. In T. S. et al (Ed.), ECAI (pp. 1077-1078). doi 10. 3233/978-1-61499-419-0-1077.

Paldi, A. (2003). Stochastic gene expression during cell differentiation: order from disorder? Cell Mol. Life Sci., 60, 1775-1779.

Papadopoulos, A., Roy, P., \& Pachet, F. (2016). Assisted lead sheet composition using flowcomposer. In M. Rueher (Ed.), Principles and Practice of Constraint Programming: 22nd International Conference (pp. 769-785). Cham: Springer. doi 10.1007/978-3-319-44953-1_48.

Rosen, R. (1991). Life itself: a comprehensive inquiry into the nature, origin, and fabrication of life. Columbia U. P.

Ruiz-Mirazo, K., Peretó, J., \& Moreno, A. (2004). A universal definition of life: Autonomy and open-ended evolution. Origins of life and evolution of the biosphere, 34, 323-346. doi:10.1023/B: ORIG.0000016440.53346.dc

Saetzler, K., Sonnenschein, C., \& Soto, A. (2011). Systems biology beyond networks: Generating order from disorder through selforganization. Seminars in Cancer Biology, 21, 165 - 174. doi 10.1016/ j.semcancer.2011.04.004.

Soros, L., \& Stanley, K. O. (2014). Identifying necessary conditions for open-ended evolution through the artificial life world of chromaria. In ALIFE 14: The Fourteenth Conference on the Synthesis and Simulation of Living Systems (pp. 793-800). volume 14. doi $10.7551 / 978-0-262-32621-6-\operatorname{ch} 128$.

Stadler, B., Stadler, P., Wagner, G., \& Fontana, W. (2001). The topology of the possible: Formal spaces underlying patterns of evolutionary change. Fournal of Theoretical Biology, 213, 241 - 274. doi $10.1006 /$ jtbi.2001.2423.

Stephan, A. (1999). Varieties of emergence. Evolution and cognition, 5, 50-59.

Tohme, M., Fini, J.-B., Laudet, V., \& Demeneix, B. (2012). Chapter 8 small model organisms as tools in food safety research. In HormoneDisruptive Chemical Contaminants in Food (pp. 136-153). The Royal Society of Chemistry. doi $10.1039 / 9781849732970-00136$.

Turing, A. M. (1950). Computing machinery and intelligence. Mind, $59,433-460$.

Turing, A. M. (1952). The chemical basis of morphogenesis. Philosophical Transactions of the Royal Society of London. Series B, Biological Sciences, 237, 37-72. doi:10.1098/rstb.1952.0012.

Varela, F., Maturana, H., \& Uribe, R. (1974). Autopoiesis: The organization of living systems, its characterization and a model. Biosystems, 5, 187 - 196. doi $10.1016 / 0303-2647$ (74)90031-8

de Vladar, H. P., Santos, M., \& Szathmáry, E. (2017). Grand views of evolution. Trends in Ecology \& Evolution, 32, 324 - 334. doi:10.1016/ j.tree.2017.01.008

Wagner, G. P., \& Lynch, V. J. (2010). Evolutionary novelties. Current Biology, 20, R48 - R52. doi $10.1016 /$ j.cub.2009.11.010

West, G., Brown, J., \& Enquist, B. (1999). The fourth dimension of life: Fractal geometry and allometric scaling of organisms. Science, 284, 1677-1679. doi $10.1126 /$ science.284.5420.1677.

West-Eberhard, M. J. (2003). Developmental plasticity and evolution. Oxford University Press.

Zhu, J., Zhang, Y.-T., Alber, M. S., \& Newman, S. A. (2010). Bare bones pattern formation: A core regulatory network in varying geometries reproduces major features of vertebrate limb development and evolution. PLOS ONE, 5, 1-11. doi 10.1371/journal.pone 0010892 . 